\title{
Hepatitis C Increases the Risk of Progression of Chronic Kidney Disease in Patients with Glomerulonephritis
}

\author{
Lama A. Noureddine $^{a}$ Sohail A. Usman ${ }^{a}$ Zhangsheng Yu ${ }^{a}$ Ranjani N. Moorthi ${ }^{a}$ \\ Sharon M. Moe ${ }^{\mathrm{a}, \mathrm{b}}$ \\ ${ }^{\mathrm{a}}$ Indiana University School of Medicine, and ${ }^{\mathrm{b}}$ Roudebush VAMC, Indianapolis, Ind., USA
}

\section{Key Words}

Hepatitis C - Chronic kidney disease - Glomerulonephritis •

Diabetic nephropathy • Dialysis · Focal segmental

glomerulosclerosis

\begin{abstract}
Background/Aims: We have shown that hepatitis $C$ does not increase the risk of developing chronic kidney disease (CKD), but it is not known if hepatitis $C$ worsens progression of existing CKD. Methods: We retrospectively identified patients with primary glomerulonephritis on biopsy over 4 years, evaluating the progression of CKD over time. Results: The cohort consisted of 111 patients: $21 \%$ were positive for hepatitis C, $61 \%$ were negative for hepatitis C and $18 \%$ were not tested. The hepatitis C-positive subjects were more likely to be African American ( $p=0.031)$, followed for fewer days ( $p=$ 0.007 ) and have diabetes and focal segmental glomerulosclerosis on biopsy ( $p<0.001$ ). Longitudinal follow-up of CKD progression using multiple creatinine measures analyzed by repeated measures ANCOVA demonstrated that patients with hepatitis $C$ had a worsening creatinine over time compared to the hepatitis C-negative and not tested groups $(p<0.001)$. By Cox hazards regression analyses, risk of death/ end-stage renal disease (ESRD) was decreased in patients who tested negative for hepatitis $C$ compared to testing positive $(0.46, \mathrm{Cl} 0.27-0.88)$, but this became nonsignificant after adjustment for mean arterial pressure and hemoglobin.
\end{abstract}

\section{KARGER}

(c) 2010 S. Karger AG, Basel

Fax +41613061234 E-Mail karger@karger.ch www.karger.com www.karger.com/ajn
Conclusion: Our results support that infection with hepatitis $C$ in patients with glomerulonephritis is associated with an increased risk of progression of CKD. Prospective studies are required to confirm these observations.

Copyright $\odot 2010$ S. Karger AG, Basel

\section{Introduction}

Recent National Health and Nutrition Examination Survey (NHANES) data demonstrates the prevalence of hepatitis $\mathrm{C}$ antibody-positive persons in the United States is $1.3-1.9 \%$, with a peak prevalence of $4.3 \%$ in persons aged 40-49 years [1]. In addition to liver manifestations of hepatitis $\mathrm{C}$, the viral infection has been linked to many extrahepatic manifestations including glomerulonephritis. Initial reports linked hepatitis $\mathrm{C}$ with membranoproliferative glomerulonephritis (MPGN) and mixed cryoglobulinemia, [2-4], but subsequent reports also demonstrated an association with other pathologies, most commonly focal sclerosing glomerulosclerosis (FSGS) [5-7].

Additional studies have revealed that the prevalence of hepatitis $\mathrm{C}$ virus (HCV) infection in developed countries is higher in patients undergoing dialysis [8] and in patients with chronic kidney disease (CKD) [9] than the general population. In our previous study, using a community hospital clinical database, we found that infection with hepatitis $\mathrm{C}$ was not associated with an increased
Sharon M. Moe, MD, Prof. of Medicine and Anatomy and Cell Biology Division of Nephrology, Vice-Chair for Research, Department of Medicine Indiana University School of Medicine

1001 W. 10th Street, OPW 526, Indianapolis, IN 46202 (USA)

Tel. +1 317278 2868, Fax +1 317278 2860, E-Mail smoe@iupui.edu 
likelihood of developing CKD [10], similar to studies using the NHANES database [11]. In an analysis using the Veterans Administration medical record system, hepatitis $C$ was also not associated with a risk of developing CKD. However, hepatitis $\mathrm{C}$ was associated with an increased likelihood of developing end-stage renal disease (ESRD) in patients with CKD stage 4 [12]. These differences highlight our incomplete understanding of the role of hepatitis C in CKD.

In the present study, we hypothesize that hepatitis $\mathrm{C}$ infection increases the rate of progression of CKD in patients with biopsy-proven glomerulonephritis, and the underlying type of renal pathology may impact this relationship. To test our hypothesis, we evaluated patients with glomerulonephritis to determine if the hepatitis $\mathrm{C}$ positivity at the time of biopsy independently determined the rate of CKD progression, ESRD or death.

\section{Methods}

Subjects were identified from billing records of all kidney biopsy reports by pathologists (Drs. Carrie Philips, Stephen Bonsib and Moo-Nahm Yum) at Indiana University between January 1, 2001 and December 31, 2004. A total of 379 biopsy reports were identified, and the final cohort consisted of all subjects who had glomerulonephritis, excluding all transplant biopsies, biopsies done on subjects on dialysis or those with an indication for biopsy of acute renal failure. 116 subjects then had additional information extracted from the electronic medical record and additional inclusion/exclusion criteria applied. Subjects must have had a creatinine at the time of biopsy, and at least one follow-up creatinine. In addition, subjects with an aspartate aminotransferase (AST) or alanine aminotransferase (ALT) level greater than $200 \mathrm{U} / 1$ were excluded to minimize any impact of acute liver toxicity on creatinine. The final cohort consisted of 111 patients that had one of the following four biopsy diagnoses: diabetic glomerulosclerosis/nephropathy (DN), FSGS, MPGN and IgA nephropathy (IgA).

Subjects were divided into three groups: hepatitis C-positive, hepatitis C-negative and hepatitis $\mathrm{C}$ not tested at any time prior to the kidney biopsy. A positive hepatitis $\mathrm{C}$ test result was defined as a positive antibody result by third generation ELISA with or without PCR confirmation. Potential confounders of the relationship between hepatitis $\mathrm{C}$ and $\mathrm{CKD}$ were evaluated by chart review of baseline data and included age, gender, race, biopsy diagnosis, use of either angiotensin-converting enzyme inhibitor (ACE-I) or angiotensin receptor blocker $(\mathrm{ARB})$, creatinine, hemoglobin $(\mathrm{Hgb}), \mathrm{HgbA1C}$, mean arterial pressure $(\mathrm{MAP}=2 / 3$ diastolic $\mathrm{BP}$ $+1 / 3$ systolic $\mathrm{BP}$ ), liver transaminases (ALT, AST), number of creatinine checks, number of overall clinic appointments and number of renal and liver clinic appointments (beginning with the biopsy date until the final creatinine available as of study endpoint). The information closest to the time of the biopsy was utilized as long as it was within 30 days before or after the biopsy, with the exception of hepatitis $C$ results, and use of ACE-I/ARB which were evaluated up to one year before or after the time of biopsy to make sure we captured the assays and use. Creatinine checks at multiple visits were evaluated for use in the longitudinal analyses, with a brief review of clinic notes and other lab tests to make sure the creatinine was not due to some acute intervention, hypotension or other clinical change. Subjects were followed until the last appointment in the clinical database, dialysis initiation, endpoint of study (December 31, 2006) or death.

\section{Statistical Analyses}

Demographic, laboratory, and biopsy results were first compared among the three hepatitis $\mathrm{C}$ cohorts (positive, negative and not tested) by ANOVA or Pearson $\chi^{2}$ tests, unless the expected counts were less than five in which a two-sided Fisher's exact test was used. To determine the association of hepatitis $\mathrm{C}$ with the risk of developing ESRD or dying, the time to event (ESRD or death) was analyzed using Kaplan-Meier estimates with statistical comparisons among hepatitis $\mathrm{C}$ status made using the log-rank test. Univariate Cox proportional hazards regression was used initially to test the relationship between hepatitis C status as well as other covariates and the outcome of ESRD or death. Hepatitis C status was then adjusted for potential cofounders (baseline creatinine or MDRD, biopsy, age, use of ACE/ARB, blood pressure and hemoglobin) in a stepwise manner in multivariate Cox proportional hazards regression models. We also evaluated the effect of hepatitis $\mathrm{C}$ status and underlying pathology by biopsy on the slope of the change (time effect on creatinine value) in creatinine using a repeated measurement ANCOVA adjusting for the covariates: baseline age, gender, race, MAP, use of ARB/ACE and hemoglobin. All the analyses were conducted using SAS 9.1.

\section{Results}

The final cohort consisted of 111 patients: 23 patients tested positive for hepatitis C (20.7\%), 68 tested negative for hepatitis $\mathrm{C}(61.3 \%)$ and 20 were not tested for hepatitis C (18\%) within one year before or after the date of the biopsy. The demographic and laboratory studies for each of these three groups are shown in table 1 . The hepatitis Cpositive subjects had higher liver transaminases (AST and ALT, $\mathrm{p}=0.010$ and 0.024 , respectively), were more likely to be African American ( $\mathrm{p}=0.031)$ and were followed for the least number of days $(\mathrm{p}=0.007)$. Otherwise, there were no significant differences between the three hepatitis groups (table 1). Out of the 111 biopsies, there were only four different histologic diagnoses: $\mathrm{DN}(\mathrm{n}=53,47.7 \%)$, FSGS $(\mathrm{n}=29,26 \%)$, MPGN $(\mathrm{n}=2,1.8 \%)$ and $\operatorname{IgA}(\mathrm{n}=27$, $24 \%)$. Out of the 23 patients with a positive hepatitis C test, 11 (48\%) had DN, 9 (39.1\%) had FSGS, 2 (8.7\%) had MPGN and $1(4.4 \%)$ had IgA. Hepatitis C-positive subjects were more likely to have DN or FSGS on biopsy $(\mathrm{p}<0.001)$.

Of the hepatitis C-positive patients, $16(69.6 \%)$ progressed to ESRD or death (15 ESRD and 1 death) and 7 $(30.4 \%)$ did not $(p=0.002)$. By univariate Cox analyses, 
Table 1. Baseline subject characteristics

\begin{tabular}{|c|c|c|c|c|c|}
\hline & $\begin{array}{l}\text { Hepatitis C- } \\
\text { positive }(n=23)\end{array}$ & $\begin{array}{l}\text { Hepatitis C- } \\
\text { negative }(n=68)\end{array}$ & $\begin{array}{l}\text { Hepatitis C } \\
\text { not tested }(n=20)\end{array}$ & Total & $\mathrm{p}$ \\
\hline Race Black & $12(52.2)$ & $14(20.6)$ & $3(15.0)$ & $29(26.1)$ & 0.031 \\
\hline Other & $2(8.7)$ & $8(11.8)$ & $2(10.0)$ & $12(10.8)$ & \\
\hline White & $9(39.1)$ & $46(67.7)$ & $15(75.0)$ & $70(63.1)$ & \\
\hline Gender, female & $5(21.7)$ & $24(35.3)$ & $8(40.0)$ & $37(33.3)$ & 0.385 \\
\hline Biopsy DN & $11(47.8)$ & $38(55.9)$ & $4(20.0)$ & $53(47.8)$ & $<0.0001$ \\
\hline FSGS & $9(39.1)$ & $15(22.1)$ & $5(25.0)$ & $29(26.1)$ & \\
\hline $\operatorname{IgA}$ & $1(4.4)$ & $15(22.1)$ & $11(55.0)$ & $27(24.3)$ & \\
\hline MPGN & $2(8.7)$ & 0 & 0 & $2(1.8)$ & \\
\hline Use of ACE or ARB & $16(69.6)$ & $54(81.8)$ & $16(80.0)$ & $86(78.9)$ & 0.459 \\
\hline $\mathrm{ALT}, \mathrm{U} / \mathrm{l}$ & $40 \pm 22$ & $26 \pm 18$ & $24 \pm 12$ & $29 \pm 19$ & 0.024 \\
\hline AST, U/l & $48 \pm 44$ & $28 \pm 16$ & $26 \pm 8$ & $32 \pm 259$ & 0.010 \\
\hline Age, years & $53.7 \pm 8.9$ & $52.3 \pm 13.7$ & $46.5 \pm 18.6$ & $51.5 \pm 14.0$ & 0.186 \\
\hline Creatinine, mg/dl & $2.8 \pm 1.3$ & $3.0 \pm 2.0$ & $1.9 \pm 1.4$ & $2.7 \pm 1.8$ & 0.052 \\
\hline MDRD eGFR & $31.7 \pm 17.4$ & $34.6 \pm 26.0$ & $59.2 \pm 39.4$ & $38.4 \pm 28.9$ & 0.001 \\
\hline $\mathrm{Hgb}, \mathrm{mg} / \mathrm{dl}$ & $11.8 \pm 2.0$ & $11.8 \pm 2.1$ & $12.2 \pm 2.3$ & $11.8 \pm 2.1$ & 0.769 \\
\hline Hbalc, \% & $6.1 \pm 0.9$ & $7.3 \pm 2.4$ & $4.9 \pm 1.1$ & $6.9 \pm 2.1$ & 0.127 \\
\hline Systolic BP, mm Hg & $144 \pm 28$ & $145 \pm 23$ & $126 \pm 15$ & $142 \pm 24$ & 0.056 \\
\hline $\mathrm{MAP}, \mathrm{mm} \mathrm{Hg}$ & $103 \pm 19$ & $99 \pm 14$ & $94 \pm 13$ & $99 \pm 15$ & 0.347 \\
\hline Cr measurements, $\mathrm{n}$ & $15.2 \pm 16.5$ & $13.7 \pm 10.4$ & $8 \pm 4.8$ & $13 \pm 11.4$ & 0.078 \\
\hline Liver clinic visits, $\mathrm{n}$ & $1.6 \pm 4.8$ & $0.6 \pm 2.7$ & $0 \pm 0$ & $0.7 \pm 3$ & 0.212 \\
\hline Nephrology clinic visits, $\mathrm{n}$ & $3.3 \pm 3.7$ & $5.4 \pm 5.3$ & $5.1 \pm 6$ & $4.9 \pm 5.2$ & 0.227 \\
\hline Days followed & $380 \pm 355$ & $588 \pm 469$ & $1,176 \pm 1,785$ & $651 \pm 882$ & 0.007 \\
\hline (median; range) & $279(1-1,195)$ & $479(1-1,545)$ & $971(10-8,286)$ & $439(1-8,286)$ & \\
\hline
\end{tabular}

Data is presented as $\mathrm{n}(\%)$ or mean $\pm \mathrm{SD}$.

baseline creatinine or MDRD, biopsy, age, use of ACE/ $\mathrm{ARB}$, blood pressure and hemoglobin were all associated with the risk of ESRD/death at $\mathrm{p} \leq 0.1$ and are included in the model. Table 2 shows the results using both creatinine and eGFR by MDRD. Being hepatitis C-negative compared to hepatitis C-positive was associated with a 0.46 reduction in risk of ESRD or death regardless of whether creatinine or eGFR by MDRD was utilized. The relationship persisted after adjustment for baseline creatinine, underlying pathology by renal biopsy, age, gender, race and use of ACE/ARB. However, the further addition of either MAP or hemoglobin increased the confidence interval and was nonsignificant with some differences when creatinine or eGFR by MDRD was used (table 2). Figure 1 shows the Kaplan-Meier curves for time to event of ESRD or death by hepatitis C status.

Multiple measures of creatinine were used to evaluate the slope of serum creatinine using all available data during the follow-up period (table 3). After adjusting for baseline creatinine, age, gender, race, MAP, use of ACE/ $A R B$ and hemoglobin, subjects who were positive for hepatitis $\mathrm{C}$ had a more rapid decline in kidney function than those who were negative or not tested (overall $p<$ 0.001). This pattern held true for all three biopsy classes (table 3). Thus, these analyses demonstrate that hepatitis C-positive patients had a faster decline in CKD (increased creatinine) regardless of the underlying pathology.

\section{Discussion}

In the present study, we tested the hypothesis that hepatitis $\mathrm{C}$ infection increases the rate of progression of CKD in patients with glomerulonephritis. Our study demonstrated that the change in creatinine using all available data during longitudinal follow-up was substantially worse for subjects with hepatitis C: an increase in serum creatinine of $1.3 \mathrm{mg} / \mathrm{dl} /$ year for hepatitis $\mathrm{C}$ subjects compared to either hepatitis $\mathrm{C}$-negative or those not tested. This risk was even greater for subjects with diabetic nephropathy. Thus, our study demonstrates that hepatitis $\mathrm{C}$ is a risk factor for progression of glomerulonephritis. 
Table 2. Cox HR of progression to ESRD or death adjusted for covariates

\begin{tabular}{lcc}
\hline $\begin{array}{l}\text { Variables and models } \\
\text { HR }(\mathrm{CI})\end{array}$ & $\begin{array}{l}\text { MDRD eGFR: } \\
\text { HR }(\mathrm{CI})\end{array}$ \\
\hline $\begin{array}{l}\text { Hepatitis } C^{*} \\
\text { Negative vs. positive }\end{array}$ & $0.46(0.27,0.88)$ & $0.46(0.27,0.88)$ \\
Not done vs. positive & $0.11(0.03,0.34)$ & $0.11(0.03,0.34)$ \\
\hline Hepatitis C plus baseline Cr* & & \\
Negative vs. positive & $0.44(0.24,0.85)$ & $0.42(0.23,0.82)$ \\
Not done vs. positive & $0.22(0.05,0.68)$ & $0.27(0.06,0.83)$ \\
\hline Hepatitis C plus Cr, biopsy, age, gender, race* & \\
Negative vs. positive & $0.39(0.20,0.81)$ & $0.41(0.20,0.85)$ \\
Not done vs. positive & $0.23(0.05,0.81)$ & $0.29(0.06,0.97)$ \\
\hline Hepatitis C plus Cr, biopsy, age, gender, race, use of ACE/ARB & \\
Negative vs. positive & $0.37(0.19,0.76)$ & $0.41(0.20,0.85)$ \\
Not done vs. positive & $0.24(0.05,0.84)$ & $0.30(0.07,1.04)$ \\
\hline
\end{tabular}

Hepatitis C plus Cr, biopsy, age, gender, race, use of $A C E / A R B$, MAP

Negative vs. positive $\quad 0.64(0.21,1.97) \quad 0.60(0.21,0.88)$

Not done vs. positive $\quad 0.27(0.01,1.89) \quad 0.27(0.01,1.75)$

Hepatitis C plus Cr, biopsy, age, gender, race, use of $A C E / A R B$, MAP, $\mathrm{Hgb}$

Negative vs. positive $\quad 0.64(0.22,1.94) \quad 0.52(0.18,1.58)$ Not done vs. positive $\quad 0.21(0.01,1.37) \quad 0.17(0.01,1.11)$

${ }^{*} \mathrm{p} \leq 0.01$ for analyses of all three groups (hepatitis C-positive, negative and not done).

Table 3. Increase in creatinine over time, $\mathrm{mg} / \mathrm{dl} / \mathrm{year}$

\begin{tabular}{lllll}
\hline & $\begin{array}{l}\text { Hep. C- } \\
\text { positive }\end{array}$ & $\begin{array}{l}\text { Hep. C- } \\
\text { negative }\end{array}$ & $\begin{array}{l}\text { Hep. C } \\
\text { not tested }\end{array}$ & $\mathrm{p}$ \\
\hline All subjects & 1.33 & 0.07 & 0.13 & $<0.001$ \\
Diabetic nephropathy & 4.70 & 0.11 & 1.72 & $<0.001$ \\
FSGS & 0.46 & 0.18 & 0.003 & 0.0023 \\
IgA nephropathy & 2.98 & 0.17 & 0.04 & $<0.043$ \\
\hline
\end{tabular}

Repeated measures ANCOVA model using multiple measures of creatinine over time, adjusted for baseline age, gender, race, MAP, use of ARB/ACE and hemoglobin.

Our results also demonstrate that hepatitis $\mathrm{C}$ positivity increased the risk of developing ESRD or death and resulted in a faster decline in kidney function over time, even after adjustments for the underlying renal pathology. The risk of death/ESRD was decreased by $50 \%$ in hepatitis C-negative patients compared to hepatitis C-posi-

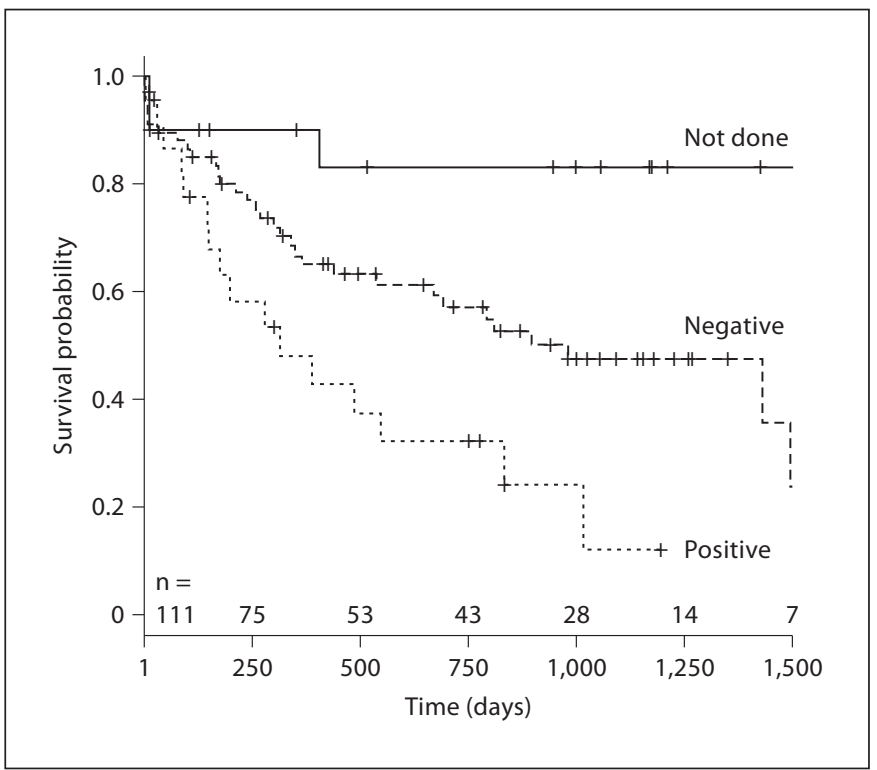

Fig. 1. Time to ESRD or death. A Kaplan-Meier plot of the proportion of subjects not reaching ESRD or dying (surviving) for each of the hepatitis $\mathrm{C}$ tested groups. Log-rank test among the three groups demonstrated $\mathrm{p}=0.0006$. The number of subjects at each time point is along the $\mathrm{x}$-axis, beginning with 111 subjects at time 0 . Not done $=$ Not tested for hepatitis $\mathrm{C}$; negative and positive $=$ results for those tested for hepatitis C.

tive patients. This difference was even greater for subjects who were not tested for hepatitis $\mathrm{C}$ compared to hepatitis $\mathrm{C}$ subjects. These results were, however, attenuated by blood pressure (in the creatinine model but not MDRD model) and hemoglobin (in both creatinine and MDRD models), which are factors that may impact the progression of CKD, although this has not been specifically tested in patients with hepatitis C. In both analyses, subjects who were not tested for hepatitis $\mathrm{C}$ did better. While higher baseline GFR is certainly one explanation, it is also possible that these individuals were considered by their physicians to be less likely to engage in risky behavior and therefore may have been more compliant with other medications and possible other behaviors such as smoking cessation, exercise and dietary compliance.

There is a higher prevalence of hepatitis $\mathrm{C}$ in CKD and dialysis patients [9], and our results suggest a worsening of CKD with hepatitis C. In our previous study [10], we showed a significant decreased risk of CKD in hepatitis $C$ patients by cross-sectional analyses after adjustment for risk factors of CKD like age, baseline eGFR, diabetes and hypertension (OR: 0.69, 95\% CI: 0.62-0.77). However, these unexpected results were no longer significant in lon- 
gitudinal analysis (HR: 0.896, 95\% CI: 0.790-1.015), demonstrating that patients with hepatitis $\mathrm{C}$ did not have a higher risk of developing CKD. Tsui et al. [12], using the NHANES database of 366 hepatitis C-positive patients, showed hepatitis $\mathrm{C}$ to have an age-dependent relationship with albuminuria, but also found a reduced risk of patients with HCV having CKD defined as an eGFR less than 60 $\mathrm{ml} / \mathrm{min} / 1.73 \mathrm{~m}^{2}$ (OR: 0.45, CI: $0.24-0.85, \mathrm{p}=0.02$ ).

A third study, evaluating 25,782 hepatitis $\mathrm{C}$ seropositive and seronegative veterans using the Veterans Affairs Puget Sound Health Care System database also found the prevalence of CKD was lower for seropositive veterans than seronegative veterans ( 4.8 vs. $6 \%$ ), but after adjusting for age, race, gender, diabetes and hypertension, seropositive patients had a significantly higher prevalence of CKD (OR: 1.40, 95\% CI: 1.11-1.76) [13]. Tsui et al. [12] in another study using the Veterans Administration database showed that patients seropositive for HCV were less likely than seronegative patients to have baseline CKD or a decline in GFR. However, seropositive patients were at a higher risk of developing ESRD and were more likely to experience a rapid decline in GFR when progression to ESRD occurred. This was prominent in patients between the ages of 18-70 years old and those with either a normal GFR or a moderately decreased GFR $(30 \mathrm{ml} / \mathrm{min}$ per $1.73 \mathrm{~m}^{2}$ ) according to the MDRD equation [14].

In the present study, we found that hepatitis $\mathrm{C}$ positivity increased the risk of death or dialysis when adjusted for baseline creatinine, biopsy pathology, age, gender, race, use of $\mathrm{ACE} / \mathrm{ARB}$ and the rate of decline in kidney function assessed by creatinine in patients with biopsyproven glomerulonephritis. These findings may explain why there is no overall association of hepatitis $C$ with CKD in the studies by Moe et al. [10] and Tsui et al. [11], but how patients with hepatitis $\mathrm{C}$ are at increased risk of developing ESRD in another study [12]. If the adverse effect of hepatitis $\mathrm{C}$ is primarily on glomerular disease progression, then mixed cohorts of both non-glomerular and glomerular diseases may fail to find a relationship. There is also biologic plausibility that hepatitis $\mathrm{C}$ may principally impact glomerular disease more, as hepatitis $\mathrm{C}$ antigens have been isolated in glomeruli and tubules on kidney biopsies [15-17]. Additional histological findings include deposition of circulating immune complexes and viral particles in the subendothelium and mesangium $[16,18-19]$. Thus, the adverse effect of hepatitis C on glomerular disease may be due to direct viral-induced damage to the glomeruli and tubules. Whether this is due to increased viral damage in areas of underlying glomerular damage or immunomodulation in hepatitis $\mathrm{C}$ that aug- ments underlying glomerular injury is not known. A recent study found that co-infection with HIV and hepatitis $\mathrm{C}$ led to an increased risk of CKD and mortality, suggesting that the immunologic mechanisms leading to glomerular disease may be worsened by hepatitis C [20].

Our study also confirmed other studies that demonstrated that most patients with hepatitis $\mathrm{C}$ have $\mathrm{DN}$ or FSGS, but not MPGN, simply because DN, FSGS and hepatitis $\mathrm{C}$ are all common diseases, especially in inner-city hospitals in the United States. The biopsy type also influenced the effect of hepatitis $C$ on the progression to ESRD or death and progression of CKD. Furthermore, the slopes of change in creatinine over time for the three biopsy groups (DN, FSGS and IgA) were all statistically significant. However, the increase in creatinine was greater in the patients with diabetic nephropathy compared to the other two histologies (FSGS and IgA nephropathy). Two other studies also found that hepatitis $C$ positivity adversely affected diabetic nephropathy [21-22]. In Japan, Soma et al. [22] examined 2,370 renal biopsies and found the highest prevalence of hepatitis C infection (19.5\%) was in patients with DN. Furthermore, the decline in renal function, defined by the slope of reciprocal serum creatinine $(1 / \mathrm{Scr})$, was significantly higher in the hepatitis $\mathrm{C}$ positive $\mathrm{DN}$ than the hepatitis $\mathrm{C}$-negative $\mathrm{DN}$ group $(\mathrm{p}=$ $0.001)$, and renal survival was worse $(p=0.019)$ [22]. In a second smaller study [21], hepatitis C positivity was found to be a significant and independent risk factor for developing ESRD in diabetic patients regardless of the presence of diabetic nephropathy, diabetes duration and initial renal function (OR: 3.49, 95\% CI: 1.27-9.57, $\mathrm{p}=0.015$ ). Our study in an urban hospital setting confirms these observations in patients with diabetic nephropathy, and extends these findings to FSGS and IgA nephropathy.

There are several limitations to our study. First, our sample size was relatively small and the data was collected retrospectively, which limited detailed information of comorbidities and severity of liver disease. Second, different hepatitis $\mathrm{C}$ assays were used during the study period and our study lacked PCR confirmatory hepatitis $\mathrm{C}$ testing for all patients. Third, possible bias may have existed as to which patients underwent a kidney biopsy and which patients were followed; patients with a higher creatinine and those who were hepatitis C-positive were probably more likely to get a biopsy. Fourth, we lacked true GFR estimates with iothalamate or other gold standard measurement techniques.

In the present study, we chose to evaluate creatinine rather than the MDRD formula. Creatinine may be artificially low with muscle wasting and altered creatinine 
metabolism in liver disease and none of the estimating equations have been validated in patients with hepatitis C. As shown in table 2, we found very little difference in our Cox model when we used MDRD rather than creatinine. However, we did find attenuation of our Cox model with blood pressure and hemoglobin and some differences between creatinine and MDRD when these parameters were added to the model. Blood pressure was only a single measurement, but blood pressure is a known risk factor for acceleration of kidney disease and the effect may mask any affect of hepatitis C. Hemoglobin also attenuated the effect of hepatitis $\mathrm{C}$. This result is more surprising given the relatively normal levels of $\mathrm{Hgb}(11.8 \mathrm{mg} /$ $\mathrm{dl}$ ) and no obvious differences between groups at baseline. The effect of hemoglobin on progression of CKD is currently controversial, especially when the hemoglobin is altered with erythropoietin-stimulating agents. Unfortunately, we did not have information on erythropoietin use.

Our study has several clinical and research implications. Current Kidney Disease Improving Global Outcome
(KDIGO) guidelines recommend HCV screening if patients fulfill criteria for having risk factors for HCV [23] for patients initiating dialysis, and possibly for patients who have evidence of glomerulonephritis [9]. Our previous data do not support routine screening for hepatitis $\mathrm{C}$ as a method to identify patients at risk for CKD [10]. However, the present study does support routine screening for hepatitis $\mathrm{C}$ in patients with underlying glomerular disease, as this may identify patients at risk of progression of CKD to ESRD or death. However, future randomized controlled trials are needed to investigate whether treating HCV may alter the progression of CKD in patients with known biopsy-proven glomerulonephritis.

\section{Acknowledgment}

This study was supported by a grant from the National Kidney Foundation of Indiana and NIH K24DK002775 (S.M.M.).

\section{References}

1 Armstrong GL, Wasley A, Simard EP, McQuillan GM, Kuhnert WL, Alter MJ: The prevalence of hepatitis $C$ virus infection in the United States, 1999 through 2002. Ann Intern Med 2006;144:705-714.

-2 Burstein DM, Rodby RA: Membranoproliferative glomerulonephritis associated with hepatitis Cvirus infection. J Am Soc Nephrol 1993;4:1288-1293.

-3 Doutrelepont JM, Adler M, Willems M, Durez $\mathrm{P}$, Yap SH: Hepatitis $\mathrm{C}$ infection and membranoproliferative glomerulonephritis. Lancet 1993;341:317.

4 Johnson RJ, Gretch DR, Yamabe H, et al: Membranoproliferative glomerulonephritis associated with hepatitis $\mathrm{C}$ virus infection. $\mathrm{N}$ Engl J Med 1993;328:465-470.

$\checkmark 5$ Gopalani A, Ahuja TS: Prevalence of glomerulopathies in autopsies of patients infected with the hepatitis $\mathrm{C}$ virus. Am J Med Sci 2001;322:57-60.

-6 Motta M, Malaguarnera M, Restuccia N, Romano M, Vinci E, Pistone G: Focal segmental glomerulosclerosis and hepatitis $\mathrm{C}$ virus: a case report. Panminerva Med 2001;43:4952.

7 Stehman-Breen C, Alpers CE, Fleet WP, Johnson RJ: Focal segmental glomerular sclerosis among patients infected with hepatitis C virus. Nephron 1999;81:37-40.

$>8$ Jadoul M, Poignet JL, Geddes C, et al: The changing epidemiology of hepatitis $\mathrm{C}$ virus (HCV) infection in haemodialysis: European multicentre study. Nephrol Dial Transplant 2004;19:904-909.
9 KDIGO clinical practice guidelines for the 16 Sansonno D, Gesualdo L, Manno C, Schena prevention, diagnosis, evaluation, and treatment of hepatitis $\mathrm{C}$ in chronic kidney disease. Kidney Int Suppl 2008;109:S1-S99.

-10 Moe SM, Pampalone AJ, Ofner S, Rosenman M, Teal E, Hui SL: Association of hepatitis C virus infection with prevalence and development of kidney disease. Am J Kidney Dis 2008;51:885-892.

11 Tsui JI, Vittinghoff E, Shlipak MG, O’Hare AM: Relationship between hepatitis $\mathrm{C}$ and chronic kidney disease: results from the Third National Health and Nutrition Examination Survey. J Am Soc Nephrol 2006;17: 1168-1174

12 Tsui JI, Vittinghoff E, Shlipak MG, et al: As sociation of hepatitis $\mathrm{C}$ seropositivity with increased risk for developing end-stage renal disease. Arch Intern Med 2007; 167:12711276.

-13 Dalrymple LS, Koepsell T, Sampson J, et al: Hepatitis $C$ virus infection and the prevalence of renal insufficiency. Clin J Am Soc Nephrol 2007;2:715-721.

14 Levey AS, Bosch JP, Lewis JB, Greene T, Rogers N, Roth D: A more accurate method to estimate glomerular filtration rate from serum creatinine: a new prediction equation Modification of Diet in Renal Disease Study Group. Ann Intern Med 1999;130:461-470.

15 Kasuno K, Ono T, Matsumori A, et al: Hepatitis $\mathrm{C}$ virus-associated tubulointerstitial injury. Am J Kidney Dis 2003;41:767-775. FP, Dammacco F: Hepatitis C virus-related proteins in kidney tissue from hepatitis $\mathrm{C}$ virus-infected patients with cryoglobulinemic membranoproliferative glomerulonephritis. Hepatology 1997;25:1237-1244.

-17 Sansonno D, Lauletta G, Montrone M, Grandaliano G, Schena FP, Dammacco F: Hepatitis $\mathrm{C}$ virus RNA and core protein in kidney glomerular and tubular structures isolated with laser capture microdissection. Clin Exp Immunol 2005;140:498-506

18 Alpers CE, Smith KD: Cryoglobulinemia and renal disease. Curr Opin Nephrol Hypertens 2008;17:243-249.

19 Martin M, Sole M: Membranoproliferative glomerulonephritis associated with hepatitis C infection. J Hepatol 2004;41:881.

20 Fischer MJ, Wyatt CM, Gordon K, et al: Hepatitis $\mathrm{C}$ and the risk of kidney disease and mortality in veterans with HIV. J Acquir Immune Defic Syndr 2010;53:222-226.

$>21$ Crook ED, Penumalee S, Gavini B, Filippova $\mathrm{K}$ : Hepatitis $\mathrm{C}$ is a predictor of poorer renal survival in diabetic patients. Diabetes Care 2005;28:2187-2191.

22 Soma J, Saito T, Taguma Y, et al: High prevalence and adverse effect of hepatitis $C$ virus infection in type II diabetic-related nephropathy. J Am Soc Nephrol 2000;11:690699.

23 Ghany MG, Strader DB, Thomas DL, Seeff LB: Diagnosis, management, and treatment of hepatitis C: an update. Hepatology 2009; 49:1335-1374. 